\title{
Primary Giant Splenic and Hepatic Echinococcal Cysts Treated by Laparoscopy
}

\author{
Oana Stanciulea, Ph.D., Mihai Adrian Eftimie, M.D., Iulian Mosteanu, M.D., Luiza Tirca, M.D., Irinel Popescu, Ph.D. \\ Center for General Surgery and Liver Transplantation "Dan Setlacec" - Fundeni Clinical Institute, Bucharest, Romania
}

Cystic echinococcosis is a zoonosis caused by the larval stage of Echinococcus granulosus. Liver and lungs are the most commonly affected organs whereas splenic infection is rare and its primary involvement occurs in less than $2 \%$ of cases. We report a case of primary giant splenic and hepatic hydatid cyst in a 28-year-old woman who was admitted for upper right quadrant pain. The abdominal ultrasonography and computed tomography showed two cystic tumors with hydatid features in liver and spleen. Total splenectomy was performed for the splenic cyst and partial pericystectomy with drainage for the liver cyst using a laparoscopic approach. One drain was kept in place for two months due to a biliary leak of about $20 \mathrm{ml} /$ day and removed afterward. The patient was discharged on postoperative day 7. Laparoscopic approach for patients with concomitant splenic and hepatic hydatidosis is a safe and effective option.

Keywords: Hydatid disease, Laparoscopic splenectomy, Spleen hydatid cyst, Laparoscopic liver surgery, Liver hydatid cyst

This is an Open Access article distributed under the terms of the Creative Commons Attribution Non-Commercial License (http:// creativecommons.org/licenses/by-nc/4.0/) which permits unrestricted non-commercial use, distribution, and reproduction in any medium, provided the original work is properly cited.
Received June 27, 2017

Revised July 25, 2017

Accepted July 28, 2017

Corresponding author

Oana Stanciulea

Center for General Surgery and Liver Transplantation "Dan Setlacec"-

Fundeni Clinical Institute, Soseaua

Fundeni, no 258, Building A, 3rd

floor, Bucharest, Romania

Tel: +40722505035

Fax: +40213180417

E-mail: oanastanciulea@yahoo.com

\section{INTRODUCTION}

Human hydatid disease is endemic in South-Eastern $\mathrm{Eu}^{-}$ rope, the Middle East, the Mediterranean countries and China and is a major health problem because of related morbidity and worldwide spreading. Liver hydatidosis is the most frequent $^{1}$ (approximately $75 \%$ of cases) $)^{2}$ followed by lung involvement (together accounting for approximately $90 \%$ of cases). ${ }^{3}$ The spleen and other organs are rarely involved. Spleen involvement can be identified in as many as $2 \sim 6 \%$ of cases. ${ }^{4} \mathrm{Si}-$ multaneous involvement of two abdominal organs is very rare.

Major progress has been made regarding the diagnosis and the medical treatment. However, surgery remains the main option for curative disease. Although several non-surgical approaches have been described (e.g. percutaneous aspiration), they have been shown to be associated with high recurrence and morbidity rates. ${ }^{5}$ Minimally invasive techniques (laparoscopy and robotic surgery) have proven feasible and safe, with high adoption rates that can be seen in endemic as well as in non-endemic areas. Since the first laparoscopic treatment of hydatid disease in $1992,{ }^{6}$ an ever-increasing number of procedures have been reported. The continuous improvements in laparoscopic instruments as well as the steady accrual of experience of the surgical teams have allowed for more complex cases to be approached by laparoscopy nowadays.

We report a case of concomitant splenic and hepatic hydatidosis which was successfully treated using a totally laparoscopic approach.

\section{CASE REPORT}

A 28-year-old female patient that presented with right up- 
per quadrant pain, nausea and weight loss was investigated with a contrast-enhanced CT scan of the abdomen. The study demonstrated two cystic lesions, located in the spleen (116/88/75 $\mathrm{mm})$ and in the liver $(102 / 68 / 65 \mathrm{~mm}$ in diameter, segments $4 \sim 8$, between the main portal branches), respectively (Fig. 1). The patient was referred to our center for further investigation and surgical management.

At admission, the patient presented moderate discomfort and intermittent pain in the right upper quadrant, mainly after meals. Clinical examination revealed a palpable mass bellow the left costal margin, with no further abnormal findings.

Blood tests showed a normal white cell count, as well as normal liver and renal function tests. Enzyme-Linked Immunosorbent Assay (ELISA) test for Echinococcus was negative.

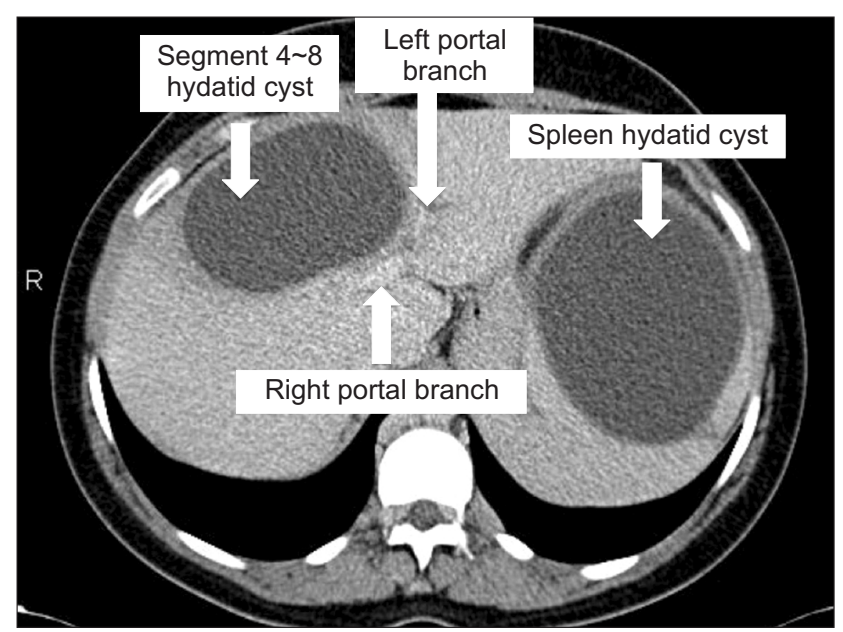

Fig. 1. Contrast enhanced computed tomography showing two intra-abdominal cystic lesions, highly suggestive for concomitant splenic and liver hydatidosis. The splenic lesion displaced most of the splenic parenchyma. The liver cyst was located in segments $4 \sim 8$ of the liver, between the main portal branches.

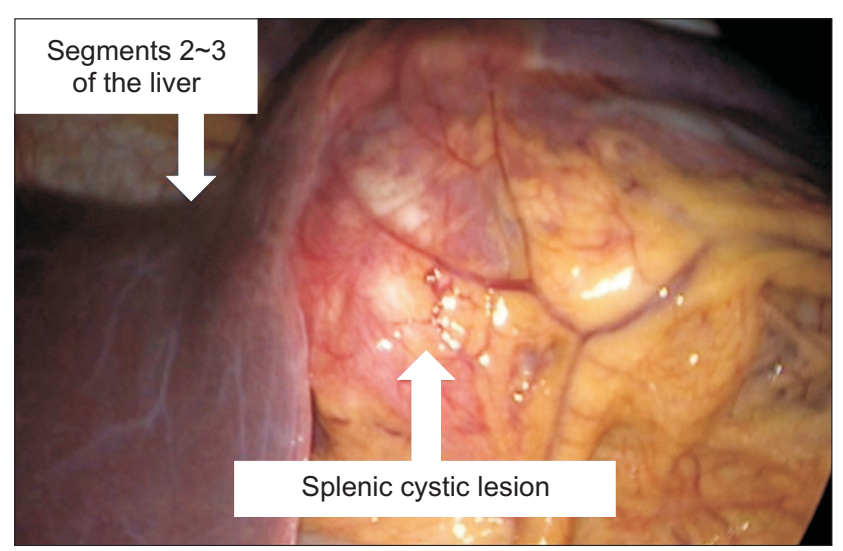

Fig. 2. Intraoperative aspect - splenic hydatic cyst.
An abdominal ultrasound scan confirmed the presence of two intra-abdominal, splenic and hepatic, cystic lesions; also a full reevaluation of the previously performed CT exam was undertaken. The radiological aspect of the cystic lesions was highly suggestive for splenic and liver hydatid disease. No other pathological findings were identified. Nevertheless, given the negative ELISA test result positive diagnosis of abdominal hydatidosis could not be established. Taking the radiological aspect into consideration, preoperative Albendazole treatment was initiated for 7 days.

Considering the important size of the splenic cyst and the remnant splenic parenchyma, the patient was prepared for a total splenectomy. Consequently, a preoperative vaccination scheme was initiated and the patient was operated one month after vaccination.

After careful evaluation and preoperative preparation, the surgical team decided to perform a single stage, totally laparoscopic splenectomy for the splenic cyst and Lagrot partial peri-cystectomy for the hepatic cyst.

The patient was placed in a partial right lateral decubitus. Pneumoperitoneum was established using a Veres needle placed through a small paraumbilical incision. the same site was used for introducing the optical trocar. Two additional ports were placed under visual control,: in the right upper quadrant and in the epigastrium, respectively. Visual inspection of the abdominal cavity confirmed the radiological findings (Fig. 2). Close adherences were identified between the splenic cystic lesion, the diaphragm and segments $2 \sim 3$ of the liver.

The lesser sac was opened near the splenic angle of the $\mathrm{Co}^{-}$ lon using monopolar cautery and LigaSure Atlas ${ }^{\circledR}$. The short gastric vessels were secured using $\mathrm{Hem}^{-}{ }^{-}-\mathrm{Lok}$ clips and $\mathrm{Li}^{-}$ gaSure Atlas. The splenic artery was identified at the superior

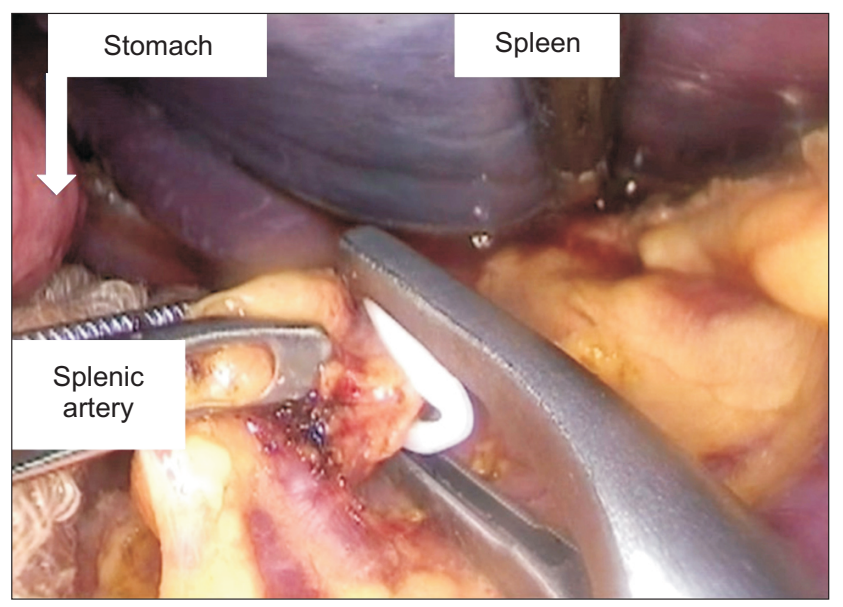

Fig. 3. Intraoperative aspect - Hem-o-Lok ${ }^{\mathrm{TM}}$ clip application on the splenic artery. 
border of the pancreatic tail. It was subsequently dissected and secured using Hem-o-Lok ${ }^{\circledR}$ clips (Fig. 3). Further dissection towards the hilum of the spleen identified the splenic vein, which was controlled by applying a white cartridge Endo GIA $^{\text {TM }}$ with Tri-Staple ${ }^{\mathrm{TM}}$ Technology (Fig. 4)

The adherences were dissected using monopolar hook and ultrasonic dissection device from Covidien ${ }^{\circledR}$. After complete mobilization of the spleen, careful hemostasis was performed.

By using a vascular first approach, the operating surgeon ensured that the risk of bleeding was reduced.

The spleen with the intact cystic lesion was inserted into an Endobag ${ }^{\circledR}$ Specimen Retrieval System and placed in the pelvic area for subsequent extraction through a Pfannensteil incision. An 18 Fr drainage tube was placed in the left flank.

Before the next step of the intervention, the patient was placed in supine anti-Trendelenburg position, slightly rotated

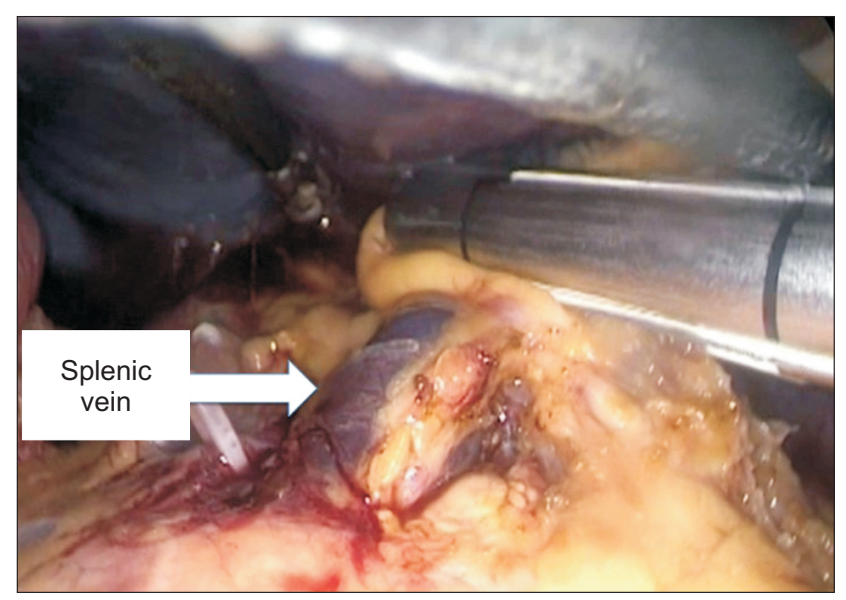

Fig. 4. Intraoperative aspect - controlling and sectioning the splenic vein with a white cartridge Endo GIA ${ }^{\mathrm{TM}}$.

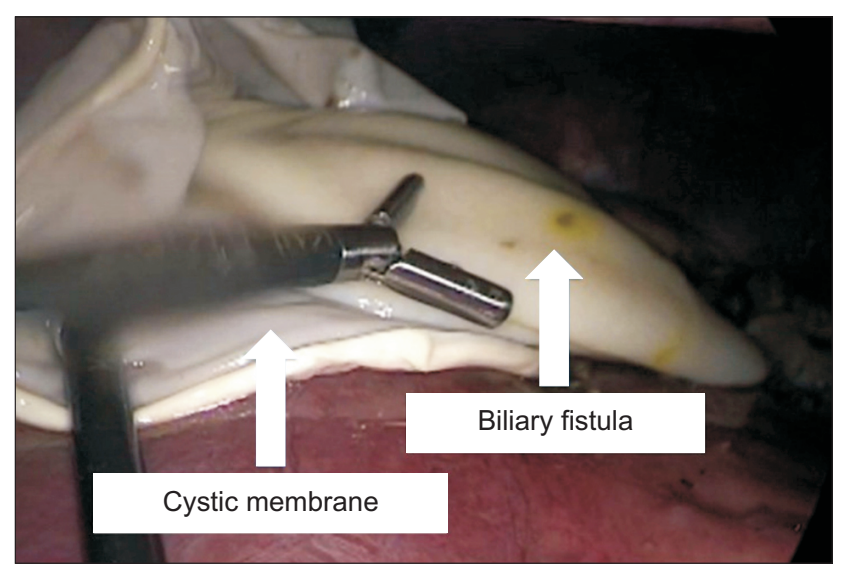

Fig. 5. Intraoperative aspect - cystic membrane with one green spot highly suggestive for a biliary fistula. to the left, in order to facilitate access to the hepatic cyst. An extra $10 \mathrm{~mm}$ trocar was placed on the mid clavicular line, seven centimeters bellow the ribs.

Gauzes soaked in scolicidal agent (1.5\% Hydrogen Peroxide) were placed around the cyst in order to limit intra-abdominal spread in case of spillage. A 5 Fr needle was placed in the cyst and $50 \mathrm{cc}$ of fluid was aspirated. A scolicidal agent (1.5\% Hydrogen Peroxide) was then perfused inside the cyst through the same needle and left for ten minutes in order to inactivate the parasites. A one-centimeter opening was performed in the peri-cyst and all of the fluid content of the cyst was aspirated with a $5 \mathrm{~mm}$ laparoscopic aspirating device. Partial peri-cystectomy was performed using ultrasonic and LigaSure ${ }^{\circledR}$ devices. The cystic membrane was inserted in a manufactured bag and placed in the pelvic area. On the surface of the membrane one green spot was identified, mark of a biliary fistula (Fig. 5) A close inspection of the remaining cavity was performed with the camera telescope but no opening in the biliary tree could be identified. Further inspection of the remnant cavity was performed after decreasing the intraabdominal pressure to $5 \mathrm{mmHg}$ in order to delineate bile leaks, as increased intraabdominal pressure can hide small bile leaks (Fig. 6).

The peri-cystic gauzes were extracted, and careful hemostasis as well as a thorough cleansing of the abdominal cavity using saline solution were performed. Two 18 Fr drainage tubes were placed inside the cystic cavity (Because thei clog harder than smaller drainage tubes).

On the $3^{\text {rd }}$ postoperative day a $20 \mathrm{cc}$ biliary fistula was revealed via one of the drainage tubes placed in the remnant cystic cavity. The other drainage tubes were taken out and the patient was discharged on the $7^{\text {th }}$ postop day. One of the $a b^{-}$ dominal drains was kept in situ for 2 months, until the biliary leak was closed, with no other complications. The patient was

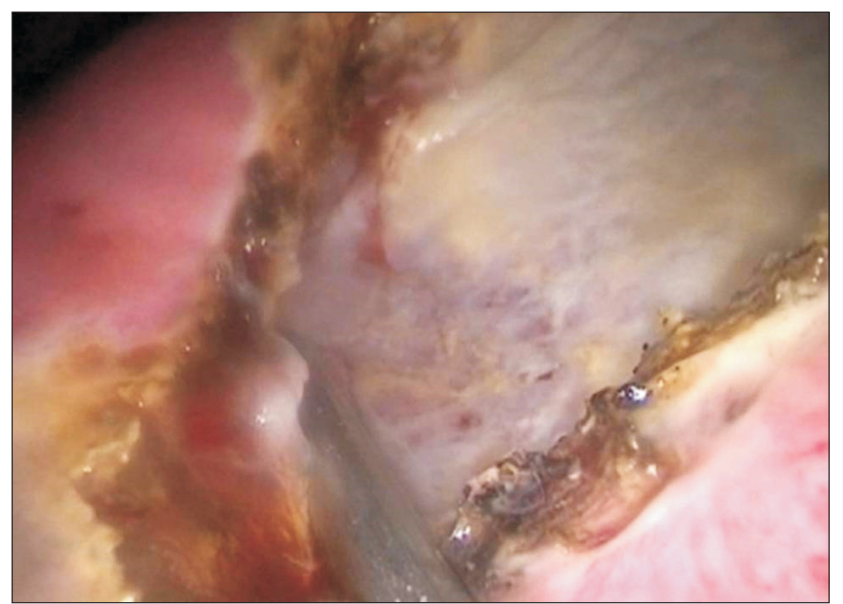

Fig. 6. Intraoperative aspect - remnant cavity with no signs of bile leak. 
started on oral Albendazole $(5 \mathrm{mg} / \mathrm{kg}$ bid) on postoperative day 1. It was administered for 4 weeks, with a pause of one week. This cycle was repeated three times.

The histopathology exam confirmed the disease.

A follow up visit six months postoperatively showed a normal WCC and a reduction of the residual cavity on USS. The patient was socially reintegrated and no signs of recurrence were present.

\section{DISCUSSION}

Several issues need to be discussed regarding the management of hydatid cysts.

The most important aspect is the choice between conservative and surgical treatment. Surgery aims to cure the disease, minimize morbidity and to prevent postoperative recurrences. Surgery is the standard treatment and regarding the size and the location of the hydatid cyst on one hand and the patients wish on the other hand, there are several methods that can be applied.

Conservative therapeutic approaches for liver hydatid cysts have been described. They aim at evacuation of cyst content, including the hydatid membrane and partial removal of the cyst such as Lagrot partial pericystectomy. Radical procedures on the oher hand aim at complete removal of the cyst with or without hepatic resection, for peripherally located cysts. Each type of approach is associated with potential specific complications: the risk of bleeding for hepatic resection and the risk of residual cavity abscess, for conservative procedures.?

Splenectomy is the most common procedure performed for splenic hydatid cysts and it is associated with low morbidity and mortality rates. Spleen-preserving surgery like partial splenectomy, cyst enucleation, deroofing of cyst with omentoplasty or external drainage are increasingly popular due to recent advances in understanding the role of the spleen in the immune response. ${ }^{8}$

Partial splenectomy was first introduced for splenic trauma and benign diseases in order to eliminate the risk of overwhelming postsplenectomy sepsis. It seems to be the procedure of choice in children and young adults because it preserves splenic immune function. ${ }^{3}$ Partial splenectomy is a technically challenging procedure and it is associated, in rare cases, with specific complications like postoperative bleeding from the spleen remnant and torsion of the splenic pedicle with secondary necrosis.

Conservative treatment - percutaneous aspiration, irrigation and re-aspiration (PAIR) CT or ultrasound-guided is associated, in very rare cases, with a risk of spillage and anaphylactic shock during the procedure and a high rate of recurrence and secondary infection on the long term. Ultrasound-guided cyst decompression is mainly indicated in patients who refuse surgery or have greater anesthetic risk. ${ }^{8}$

A further challenge arising for the surgeon is to choose between the open and the laparoscopic approach. Initially there were some concerns regarding an increased risk of intraoperative spillage of the cystic content associated with laparoscopic surgery. A proper preoperative imaging evaluation (enhanced CT exam) can describe with great accuracy the localization of the hydatid disease, rule out any other infected sites and help the surgeon decide on the best approach.

The advantages of laparoscopy also apply to this pathology and are described and highlighted in many reports. The current data, that resulting from large series and reviews, suggests that the aparoscopic approach is safe in selected patients, but is unclear whether the laparoscopic approach will ever surpass the traditional open approach and become the gold standard of treatment for selected hyadatid cysts. ${ }^{2}$ Most authors seem to agree that the size of the hydatid cyst should not influence or limit the indication for the laparoscopic approach. The exclusion criteria were cysts located in segments 1 or 7 of the liver and evidence of intrabiliary rupture.,

Proper selection of the cases can reduce intra-operative morbidity, recurrence and conversion rates to a minimum.

Surgery should be preceded by oral treatment with Albendazole (administered preoperatively for five to seven days) and in the case of total splenectomy by a proper vaccination scheme. Intraoperative chemical sterilization using various scolicidal agents must be considered as well. Great care must be taken in limiting the intra-abdominal contamination during the surgical procedure. Gauzes soaked in scolicidal agent must be placed near the cyst, in order to limit abdominal contamination.

Surgical treatment should be followed by administration of Albendazole for a minimum of three months.

Laparoscopic splenectomy for hydatid disease is a technically a difficult demanding procedure. Usually there are significant perisplenic adhesions and local inflammation. Bleeding can occur during the lysis of adhesions, which is why it is recommended to approach the vessels in the splenic hilum first, whenever possible. In cases of difficult splenectomy or when a partial splenectomy is planned, a robotic approach can be useful. The advantages offered by the robotic system (better visualization and maneuverability of the instruments) result in a better dissection of the splenic vessels and better identification of the vascularization of the future splenic remnant in partial splenectomy. ${ }^{3}$

Another important aspect is the fact that although a benign condition, hydatid disease is associated with significant morbidity. Outcomes of surgery in endemic areas reported complication rates around $30 \%$. 
The most serious complication is intra-biliary rupture witch can lead to fistula, infection and secondary biliary cirrhosis. In some cases, including ours, intra-biliary rupture is occult and only discovered during surgery. ${ }^{10}$

Theoretically the laparoscopic approach, due to magnification, can better help identify the bilio-cystic communication. In these cases a suture or a clip can be used to close the opening. ${ }^{1}$ Although in our case a green spot was clearly visible on the cystic membrane, we failed to identify the communication, probably due to the small flow of the leakage ( $20 \mathrm{cc}$ per day).

The laparoscopic approach is slowly gaining acceptance for the treatment of hydatid disease. The right procedure and the right approach should be individualized for each case.

The laparoscopic approach is a safe and effective option in patients with concomitant splenic and hepatic hydatidosis.

\section{REFERENCES}

1) Tomus $C$, Zaharie F, Mocan $L$, et al. Minimal invasive treatment of abdominal multiorgan echinococcosis. Int Surg 2013;98:61-64.

2) Tuxun T, Zhang JH, Zhao JM, et al. World review of laparoscopic treatment of liver cystic echinococcosis--914 patients. Int J Infect Dis 2014;24:43-50.

3) Vasilescu C, Tudor S, Popa M, Tiron A, Lupescu I. Robotic partial splenectomy for hydatid cyst of the spleen. Langenbecks Arch Surg 2010;395:1169-1174.

4) Jabbari Nooghabi A, Raoufian K, Motie MR. Concomitant splenic and hepatic hydatidosis: report of two cases and review of the literature. Acta Med Iran 2015;53:74-77.

5) Khoury G, Abiad F, Geagea T, Nabout G, Jabbour S. Laparoscopic treatment of hydatid cysts of the liver and spleen. Surg Endosc 2000;14:243-245.

6) Katkhouda N, Fabiani P, Benizri E, Mouiel J. Laser resection of a liver hydatid cyst under videolaparoscopy. Br J Surg 1992;79:560561.

7) Anand S, Rajagopalan S, Mohan R. Management of liver hydatid cysts - Current perspectives. Med J Armed Forces India 2012;68:304-309.

8) Rasheed K, Zargar SA, Telwani AA. Hydatid cyst of spleen: a diagnostic challenge. N Am J Med Sci 2013;5:10-20.

9) Zaharie F, Bartos D, Mocan L, Zaharie R, Iancu C, Tomus C. Open or laparoscopic treatment for hydatid disease of the liver? A 10-year single-institution experience. Surg Endosc 2013;27:21102116.

10) Zeybek N, Dede H, Balci D, et al. Biliary fistula after treatment for hydatid disease of the liver: when to intervene. World J Gastroenterol 2013;19:355-361. 